\title{
Food Frequency Questionnaire
}

(English version)

\section{General instructions}

This questionnaire refers to your eating habits during the previous year prior to the diagnosis of disease. Please answer the questions as best you can. If you are unsure of the answer, make an estimation. An estimated response is better than an empty space. Blacken the corresponding box (circle) to indicate how often, on average, you have eaten the specified food. If an error occurs write "NO" next to the wrong signs and then blacken the correct answer. Enter an answer on every line.

For any questions feel free to ask.

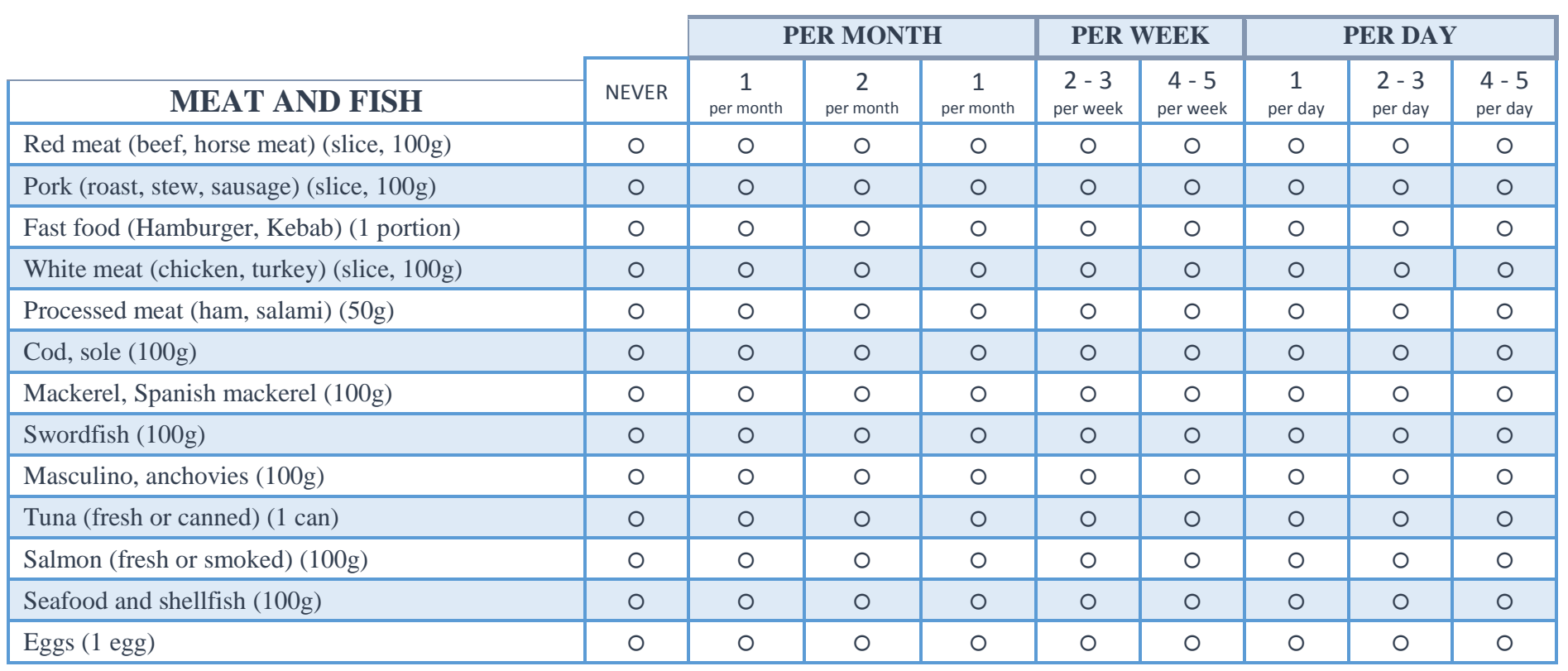

\begin{tabular}{|l|c|c|c|c|c|c|c|c|c|}
\cline { 3 - 10 } \multicolumn{2}{c|}{} & \multicolumn{3}{c|}{ PER MONTH } & \multicolumn{2}{c|}{ PER WEEK } & \multicolumn{3}{c|}{ PER DAY } \\
\cline { 3 - 11 } \multicolumn{1}{c|}{ SWEETS AND SNACKS } & NEVER & $\begin{array}{c}1 \\
\text { per month }\end{array}$ & $\begin{array}{c}2 \\
\text { per month }\end{array}$ & $\begin{array}{c}1 \\
\text { per month }\end{array}$ & $\begin{array}{c}2-3 \\
\text { per week }\end{array}$ & $\begin{array}{c}4-5 \\
\text { per week }\end{array}$ & $\begin{array}{c}1 \\
\text { per day }\end{array}$ & $\begin{array}{c}2-3 \\
\text { per day }\end{array}$ & $\begin{array}{c}4-5 \\
\text { per day }\end{array}$ \\
\hline Various pastries (3 cookies/2 pastries/1 snack) & $\circ$ & $\circ$ & $\circ$ & $\circ$ & $\circ$ & $\circ$ & $\circ$ & $\circ$ & $\circ$ \\
\hline Ice cream or slush (1 glass/cup) & $\circ$ & $\circ$ & $\circ$ & $\circ$ & $\circ$ & $\circ$ & $\circ$ & $\circ$ & 0 \\
\hline Milk chocolate (4 squares) & $\circ$ & $\circ$ & $\circ$ & $\circ$ & $\circ$ & $\circ$ & $\circ$ & $\circ$ & $\circ$ \\
\hline Dark chocolate (4 squares) & $\circ$ & $\circ$ & $\circ$ & $\circ$ & $\circ$ & $\circ$ & $\circ$ & $\circ$ & $\circ$ \\
\hline Chips or other salty snacks (1 package) & $\circ$ & $\circ$ & $\circ$ & $\circ$ & $\circ$ & $\circ$ & $\circ$ & $\circ$ & $\circ$ \\
\hline Chestnuts (6 chestnuts) & $\circ$ & $\circ$ & $\circ$ & $\circ$ & $\circ$ & $\circ$ & $\circ$ & $\circ$ & $\circ$ \\
\hline Peanuts(10 peanuts) & $\circ$ & $\circ$ & $\circ$ & $\circ$ & $\circ$ & $\circ$ & $\circ$ & $\circ$ & $\circ$ \\
\hline Pistachios (10 pistachios) & $\circ$ & $\circ$ & $\circ$ & $\circ$ & $\circ$ & $\circ$ & $\circ$ & $\circ$ & $\circ$ \\
\hline Walnuts (5 walnuts) & $\circ$ & $\circ$ & $\circ$ & $\circ$ & $\circ$ & $\circ$ & $\circ$ & $\circ$ & $\circ$ \\
\hline Almonds (10 almonds) & $\circ$ & $\circ$ & $\circ$ & $\circ$ & $\circ$ & $\circ$ & $\circ$ & $\circ$ & $\circ$ \\
\hline Hazelnuts (10 hazelnuts) & $\circ$ & $\circ$ & $\circ$ & $\circ$ & $\circ$ & $\circ$ & $\circ$ & $\circ$ & $\circ$ \\
\hline Jam, marmalade, honey (2 teaspoons) & $\circ$ & $\circ$ & $\circ$ & $\circ$ & $\circ$ & $\circ$ & $\circ$ & $\circ$ & $\circ$ \\
\hline Spreads (nutella) (2 teaspoons) & $\circ$ & $\circ$ & $\circ$ & $\circ$ & $\circ$ & $\circ$ & $\circ$ & $\circ$ & $\circ$ \\
\hline
\end{tabular}

\begin{tabular}{|l|c|c|c|c|}
\cline { 2 - 4 } \multicolumn{1}{c|}{ DRESSING } & \multicolumn{4}{c|}{ Frequencies } \\
\hline Salt added during cooking & $\circ$ NEVER & $\circ$ sometimes & $\circ$ often & $\circ$ always \\
\hline Salt added after cooking & $\circ$ NEVER & $\circ$ sometimes & $\circ$ often & $\circ$ always \\
\hline Sugar added to coffee or tea & $\circ$ NEVER & $\circ$ half teaspoon & $\circ 1$ teaspoon & $\circ$ teaspoons \\
\hline Sweeteners & $\circ$ NEVER & $\circ 1$ drop/tablet & $\circ 2$ drops/tablets & - \\
\hline Olive oil (1 spoon) & $\circ$ NEVER & $\circ$ less than 1 time per day & $\circ 1$ time per day & $\circ 2$ or more times per day \\
\hline Seed oil (1 spoon) & $\circ$ NEVER & $\circ$ less than 1 time per day & $\circ 1$ time per day & $\circ 2$ or more times per day \\
\hline
\end{tabular}


FOR SEASONAL FRUIT AND VEGETABLES, INDICATE THE CONSUMPTION IN THE SEASON

\begin{tabular}{|c|c|c|c|c|c|c|c|c|c|}
\hline \multirow[b]{2}{*}{ VEGETABLE } & \multirow[b]{2}{*}{ NEVER } & \multicolumn{3}{|c|}{ PER MONTH } & \multicolumn{2}{|c|}{ PER WEEK } & \multicolumn{3}{|c|}{ PER DAY } \\
\hline & & $\begin{array}{c}1 \\
\text { per month }\end{array}$ & $\underset{\text { per month }}{2}$ & $\begin{array}{c}1 \\
\text { per month }\end{array}$ & $\begin{array}{c}2-3 \\
\text { per week }\end{array}$ & $\begin{array}{c}4-5 \\
\text { per week }\end{array}$ & $\begin{array}{c}1 \\
\text { per day }\end{array}$ & $\begin{array}{l}2-3 \\
\text { per day }\end{array}$ & $\begin{array}{l}4-5 \\
\text { per day }\end{array}$ \\
\hline Carrot (1 carrot) & 0 & 0 & 0 & 0 & 0 & 0 & 0 & 0 & 0 \\
\hline Spinach $(120 \mathrm{~g})$ & 0 & 0 & 0 & 0 & 0 & 0 & 0 & 0 & 0 \\
\hline Broccoli (100g) (SEASON) & 0 & 0 & 0 & 0 & 0 & 0 & 0 & 0 & 0 \\
\hline Sprouts, Bruxell sprouts (100g) (SEASON) & 0 & 0 & 0 & 0 & 0 & 0 & 0 & 0 & 0 \\
\hline Cauliflower (70g) (SEASON) & 0 & 0 & 0 & 0 & 0 & 0 & 0 & 0 & 0 \\
\hline Peas $(100 \mathrm{~g})$ & 0 & 0 & 0 & 0 & 0 & 0 & 0 & 0 & 0 \\
\hline Green beans $(150 \mathrm{~g})(\mathrm{SEASON})$ & 0 & 0 & 0 & 0 & 0 & 0 & 0 & 0 & 0 \\
\hline Zucchini (100g) & 0 & 0 & 0 & 0 & 0 & 0 & 0 & 0 & 0 \\
\hline Chicory $(120 \mathrm{~g})$ & 0 & 0 & o & 0 & o & 0 & o & O & 0 \\
\hline Asparagus (100g) (SEASON) & 0 & 0 & 0 & 0 & 0 & 0 & 0 & 0 & 0 \\
\hline Onion (4 slices) & 0 & 0 & 0 & 0 & 0 & 0 & 0 & 0 & 0 \\
\hline Garlic (1 clove) & 0 & 0 & 0 & 0 & 0 & 0 & 0 & 0 & 0 \\
\hline Mushrooms (150g) (SEASON) & O & O & ○ & O & O & O & O & 0 & O \\
\hline Green salad, lettuce $(100 \mathrm{~g})$ & $\mathrm{O}$ & 0 & 0 & 0 & 0 & 0 & 0 & 0 & 0 \\
\hline Tomatoes $(100 \mathrm{~g})$ & 0 & 0 & 0 & 0 & 0 & 0 & 0 & 0 & 0 \\
\hline Fennel (half) & 0 & 0 & O & O & 0 & 0 & 0 & 0 & 0 \\
\hline Corn $(100 \mathrm{~g})$ & 0 & 0 & 0 & 0 & 0 & 0 & 0 & 0 & 0 \\
\hline Beans (100g) & 0 & 0 & 0 & 0 & 0 & 0 & 0 & 0 & 0 \\
\hline Chickpeas $(100 \mathrm{~g})$ & 0 & 0 & 0 & 0 & 0 & 0 & 0 & 0 & 0 \\
\hline Lentils $(100 \mathrm{~g})$ & 0 & 0 & 0 & 0 & 0 & 0 & 0 & 0 & 0 \\
\hline Fava beans $(100 \mathrm{~g})$ & 0 & 0 & 0 & 0 & 0 & 0 & $\mathrm{o}$ & 0 & 0 \\
\hline Artichokes ( 1 artichoke) (SEASON) & 0 & 0 & 0 & 0 & 0 & 0 & 0 & 0 & 0 \\
\hline Soy, veggie burgers, tofu (100g) & $\circ$ & 0 & O & 0 & 0 & 0 & 0 & 0 & 0 \\
\hline Pepperoni (1 pepper) & 0 & 0 & 0 & 0 & 0 & 0 & 0 & 0 & 0 \\
\hline Eggplant (half eggplant) & $\circ$ & O & ○ & O & O & O & O & 0 & O \\
\hline Green olives (4 olives) & 0 & 0 & 0 & 0 & 0 & 0 & 0 & 0 & 0 \\
\hline Black olives (4 olives) & 0 & 0 & 0 & 0 & 0 & 0 & 0 & 0 & 0 \\
\hline
\end{tabular}

\begin{tabular}{|c|c|c|c|c|c|c|c|c|c|}
\hline \multirow[b]{2}{*}{ FRUIT } & \multirow[b]{2}{*}{ NEVER } & \multicolumn{3}{|c|}{ PER MONTH } & \multicolumn{2}{|c|}{ PER WEEK } & \multicolumn{3}{|c|}{ PER DAY } \\
\hline & & $\begin{array}{c}1 \\
\text { per month }\end{array}$ & $\underset{\text { per month }}{2}$ & $\begin{array}{c}1 \\
\text { per month }\end{array}$ & $\begin{array}{c}2-3 \\
\text { per wwek }\end{array}$ & $\begin{array}{c}4-5 \\
\text { per wwek }\end{array}$ & $\begin{array}{c}1 \\
\text { per day }\end{array}$ & $\begin{array}{l}2-3 \\
\text { per day }\end{array}$ & $\begin{array}{l}4-5 \\
\text { per day }\end{array}$ \\
\hline Apple (1 fruit) & 0 & o & O & 0 & O & 0 & 0 & o & 0 \\
\hline Pear (1 fruit) & 0 & 0 & 0 & 0 & 0 & 0 & 0 & 0 & 0 \\
\hline Citrus fruits (1 fruit) (SEASON) & 0 & 0 & 0 & 0 & 0 & 0 & 0 & 0 & 0 \\
\hline Blood orange (1 fruit) (SEASON) & 0 & 0 & 0 & 0 & 0 & 0 & 0 & 0 & 0 \\
\hline Banana (1 fruit) & 0 & 0 & 0 & 0 & 0 & 0 & 0 & 0 & 0 \\
\hline Grapes (6 grains) (SEASON) & 0 & 0 & 0 & 0 & 0 & 0 & 0 & 0 & 0 \\
\hline Melon (1 slice) (SEASON) & 0 & 0 & 0 & 0 & 0 & 0 & 0 & 0 & 0 \\
\hline Watermelon (1 slice) (SEASON) & O & 0 & O & O & 0 & O & O & O & O \\
\hline Peaches (1 fruit) (SEASON) & O & O & O & O & O & O & O & O & O \\
\hline Apricots (1 fruit) (SEASON) & 0 & 0 & 0 & 0 & 0 & 0 & 0 & 0 & 0 \\
\hline Strawberries (4 strawberries) (SEASON) & O & O & O & O & O & O & O & O & O \\
\hline Kiwi (1 fruit) (SEASON) & 0 & 0 & 0 & 0 & 0 & 0 & 0 & 0 & 0 \\
\hline Cherries (6 cherries) (SEASON) & 0 & 0 & 0 & 0 & 0 & 0 & 0 & 0 & 0 \\
\hline Red fruits (SEASON) & 0 & 0 & 0 & 0 & 0 & 0 & 0 & 0 & 0 \\
\hline Pomegranate (1 fruit) (SEASON) & O & O & O & O & O & O & O & O & O \\
\hline Prickly pear (1 fruit) (SEASON) & 0 & 0 & 0 & O & 0 & 0 & 0 & 0 & 0 \\
\hline Prunes, plums (1 fruit) (SEASON) & O & O & O & O & O & O & O & 0 & O \\
\hline Figs (2 figs) (SEASON) & 0 & 0 & 0 & 0 & 0 & 0 & 0 & 0 & 0 \\
\hline
\end{tabular}




\begin{tabular}{|c|c|c|c|c|c|c|c|c|c|}
\hline \multirow[b]{2}{*}{ DRINKS } & \multirow[b]{2}{*}{ NEVER } & \multicolumn{3}{|c|}{ PER MONTH } & \multicolumn{2}{|c|}{ PER WEEK } & \multicolumn{3}{|c|}{ PER DAY } \\
\hline & & $\begin{array}{c}1 \\
\text { per month }\end{array}$ & $\begin{array}{c}2 \\
\text { per month }\end{array}$ & $\begin{array}{c}1 \\
\text { per month }\end{array}$ & $\begin{array}{c}2-3 \\
\text { per week }\end{array}$ & $\begin{array}{c}4-5 \\
\text { per week }\end{array}$ & $\begin{array}{c}1 \\
\text { per day }\end{array}$ & $\begin{array}{l}2-3 \\
\text { per day }\end{array}$ & $\begin{array}{l}4-5 \\
\text { per day }\end{array}$ \\
\hline Black tea or herbal tea (1 cup) (without fruit) & o & o & o & o & o & 0 & o & o & o \\
\hline Green tea & 0 & 0 & 0 & 0 & 0 & 0 & 0 & 0 & 0 \\
\hline Tea, herbal teas or fruit infusions & 0 & 0 & 0 & 0 & 0 & 0 & 0 & 0 & 0 \\
\hline Coffee (espresso) or cappuccino (1 cup) & 0 & 0 & 0 & 0 & 0 & 0 & 0 & 0 & 0 \\
\hline Coffee (espresso) or cappuccino decaffeinated (1 cup) & 0 & 0 & 0 & 0 & 0 & 0 & 0 & 0 & 0 \\
\hline Red wine (half glass) & 0 & 0 & 0 & 0 & 0 & 0 & 0 & 0 & 0 \\
\hline White wine (half glass) & o & o & o & o & o & O & o & o & o \\
\hline Beer $(1$ can $)$ & 0 & O & O & 0 & o & 0 & o & O & 0 \\
\hline Liqueurs (1 shot) & o & o & o & o & o & O & o & o & o \\
\hline Soft drinks (1 glass) & 0 & 0 & 0 & 0 & 0 & 0 & 0 & 0 & 0 \\
\hline Juices or smoothies (fresh fruit) & o & o & o & o & o & O & o & o & o \\
\hline Juices or smoothies (confectioned) & 0 & 0 & 0 & 0 & 0 & 0 & 0 & 0 & 0 \\
\hline Water (glasses a day) & $\begin{array}{c}\leq 3 \\
(600 \mathrm{ml})\end{array}$ & 4 & 5 & $\begin{array}{c}6 \\
(1,2 \text { It })\end{array}$ & 7 & $\begin{array}{c}8 \\
(1,6 \mid t)\end{array}$ & 9 & 10 & $\begin{array}{c}\geq 11 \\
(>2 \mathrm{It})\end{array}$ \\
\hline
\end{tabular}

\begin{tabular}{|c|c|c|c|c|c|c|c|c|c|}
\hline \multirow[b]{2}{*}{ CEREALS AND BREADS } & \multirow[b]{2}{*}{ NEVER } & \multicolumn{2}{|c|}{ PER MONTH } & \multicolumn{3}{|c|}{ PER WEEK } & \multicolumn{3}{|c|}{ PER DAY } \\
\hline & & $\begin{array}{c}1 \\
\text { per }\end{array}$ & $\begin{array}{c}2 \\
\text { per month }\end{array}$ & $\begin{array}{c}1 \\
\text { per }\end{array}$ & $\begin{array}{c}2-3 \\
\text { per week }\end{array}$ & $\begin{array}{c}4-5 \\
\text { per week }\end{array}$ & $\begin{array}{c}1 \\
\text { per day }\end{array}$ & $\begin{array}{l}2-3 \\
\text { per day }\end{array}$ & $\begin{array}{l}4-5 \\
\text { per day }\end{array}$ \\
\hline Bread and bakery products (biscuits, crackers) $(100 \mathrm{~g})$ & ○ & O & O & 0 & ○ & ○ & O & O & ○ \\
\hline Bread and flour products whole wheat $(100 \mathrm{~g})$ & 0 & O & O & 0 & 0 & O & O & O & O \\
\hline Corn flakes, wheat cereal (30g) & O & O & o & 0 & o & O & O & 0 & O \\
\hline Rice $(80 \mathrm{~g})$ & ○ & 0 & O & 0 & ○ & ○ & ○ & $\circ$ & O \\
\hline Pasta $(80 \mathrm{~g})$ & o & o & O & 0 & o & o & o & 0 & 0 \\
\hline Rice or pasta whole wheat $(80 \mathrm{~g})$ & O & 0 & 0 & 0 & O & o & 0 & 0 & 0 \\
\hline French fries $(100 \mathrm{~g})$ & ○ & 0 & O & O & ○ & ○ & ○ & O & O \\
\hline Potatoes: boiled, baked, mashed $(100 \mathrm{~g})$ & 0 & 0 & 0 & 0 & 0 & 0 & 0 & 0 & 0 \\
\hline Pizza (half pizza) & 0 & O & 0 & 0 & 0 & 0 & 0 & 0 & O \\
\hline Street food (arancini, cipolline) & o & 0 & o & 0 & o & o & o & o & 0 \\
\hline
\end{tabular}

\begin{tabular}{|l|c|c|c|c|c|c|c|c|c|}
\cline { 3 - 10 } \multicolumn{1}{c|}{} & \multicolumn{3}{c|}{ PER MONTH } & \multicolumn{2}{c|}{ PER WEEK } & \multicolumn{3}{c|}{ PER DAY } \\
\cline { 2 - 10 } \multicolumn{1}{c|}{ DAIRY } & \multirow{2}{*}{ NEVER } & $\begin{array}{c}1 \\
\text { per month }\end{array}$ & $\begin{array}{c}2 \\
\text { per month }\end{array}$ & $\begin{array}{c}1 \\
\text { per month }\end{array}$ & $\begin{array}{c}2-3 \\
\text { per week }\end{array}$ & $\begin{array}{c}4-5 \\
\text { per week }\end{array}$ & $\begin{array}{c}1 \\
\text { per day }\end{array}$ & $\begin{array}{c}2-3 \\
\text { per day }\end{array}$ & $\begin{array}{c}4-5 \\
\text { per day }\end{array}$ \\
\hline Milk (1 cup, 250ml) & $\circ$ & $\circ$ & $\circ$ & $\circ$ & $\circ$ & $\circ$ & $\circ$ & 0 & $\circ$ \\
\hline Soy milk, rice milk (1 cup, 250ml) & $\circ$ & $\circ$ & $\circ$ & $\circ$ & $\circ$ & $\circ$ & $\circ$ & $\circ$ & $\circ$ \\
\hline Yogurt (1 cup) & $\circ$ & $\circ$ & $\circ$ & $\circ$ & $\circ$ & $\circ$ & $\circ$ & $\circ$ & $\circ$ \\
\hline Yellow/hard cheese (50g) & $\circ$ & $\circ$ & $\circ$ & $\circ$ & $\circ$ & $\circ$ & $\circ$ & $\circ$ & $\circ$ \\
\hline Cottage cheese, soft cheese low fat (50g) & $\circ$ & $\circ$ & $\circ$ & $\circ$ & $\circ$ & $\circ$ & $\circ$ & $\circ$ & $\circ$ \\
\hline Mozzarella (1 mozzarella) & $\circ$ & $\circ$ & $\circ$ & $\circ$ & $\circ$ & $\circ$ & $\circ$ & $\circ$ & $\circ$ \\
\hline Cream cheese (Philadelfia) (30g) & $\circ$ & $\circ$ & $\circ$ & $\circ$ & $\circ$ & $\circ$ & $\circ$ & $\circ$ & $\circ$ \\
\hline Butter (10g) & $\circ$ & $\circ$ & $\circ$ & $\circ$ & $\circ$ & $\circ$ & $\circ$ & $\circ$ & $\circ$ \\
\hline Margarine (10g) & $\circ$ & $\circ$ & $\circ$ & $\circ$ & $\circ$ & $\circ$ & $\circ$ & $\circ$ & $\circ$ \\
\hline
\end{tabular}

\begin{tabular}{|l|c|c|c|c|c|c|c|c|c|}
\cline { 3 - 9 } \multicolumn{2}{c|}{} & \multicolumn{3}{c|}{ PER MONTH } & \multicolumn{2}{c|}{ PER WEEK } & \multicolumn{3}{c|}{ PER DAY } \\
\cline { 3 - 10 } \multicolumn{1}{c|}{ Dietary supplements and herbs } & NEVER & $\begin{array}{c}1 \\
\text { per month }\end{array}$ & $\begin{array}{c}2 \\
\text { per month }\end{array}$ & $\begin{array}{c}1 \\
\text { per month }\end{array}$ & $\begin{array}{c}2-3 \\
\text { per week }\end{array}$ & $\begin{array}{c}4-5 \\
\text { per week }\end{array}$ & $\begin{array}{c}1 \\
\text { per day }\end{array}$ & $\begin{array}{c}2-3 \\
\text { per day }\end{array}$ & $\begin{array}{c}4-5 \\
\text { per day }\end{array}$ \\
\hline Name and type: & 0 & 0 & 0 & 0 & 0 & 0 & 0 & 0 & 0 \\
\hline Name and type: & 0 & 0 & 0 & 0 & 0 & 0 & 0 & 0 & 0 \\
\hline
\end{tabular}




\section{Questionario di frequenza alimentare (versione italiana)}

\section{Istruzioni generali}

Il presente questionario si riferisce alle sue abitudini alimentari durante l'arco dell'anno precedente alla diagnosi di patologia. Risponda alle domande nel miglior modo possibile. Se non è sicura della risposta, faccia una stima. Una risposta stimata è meglio di uno spazio vuoto. Annerisca la casella relativa (cerchio) alla risposta che vuole indicare. In caso di errore scriva "NO" accanto alla risposta sbagliata e segni quella corretta. Inserisca una risposta per riga.

Per qualsiasi dubbio sulle porzioni siamo a sua disposizione per chiarimenti.

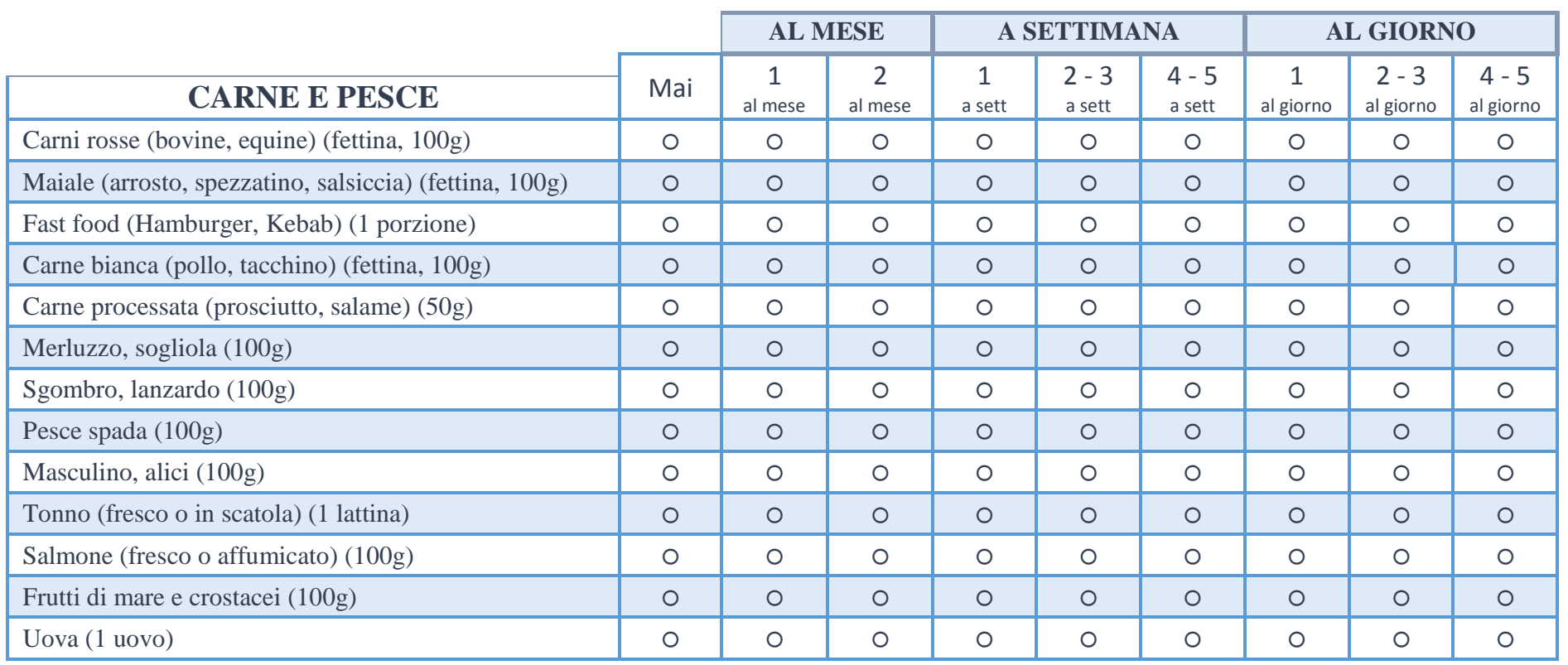

\begin{tabular}{|l|c|c|c|c|c|c|c|c|c|}
\cline { 3 - 10 } \multicolumn{2}{c|}{} & \multicolumn{2}{c|}{ AL MESE } & \multicolumn{3}{c|}{ A SETTIMANA } & \multicolumn{3}{c|}{ AL GIORNO } \\
\cline { 3 - 11 } \multicolumn{1}{c|}{ DOLCI E SNACK } & Mai & $\begin{array}{c}1 \\
\text { al mese }\end{array}$ & $\begin{array}{c}2 \\
\text { al mese }\end{array}$ & $\begin{array}{c}1 \\
\text { a sett }\end{array}$ & $\begin{array}{c}2-3 \\
\text { a sett }\end{array}$ & $\begin{array}{c}4-5 \\
\text { a sett }\end{array}$ & $\begin{array}{c}1 \\
\text { al giorno }\end{array}$ & $\begin{array}{c}2-3 \\
\text { al giorno }\end{array}$ & $\begin{array}{c}4-5 \\
\text { al giorno }\end{array}$ \\
\hline Dolci vari (3 biscotti/2 pasticcini/1 snack) & $\circ$ & $\circ$ & $\circ$ & $\circ$ & $\circ$ & $\circ$ & $\circ$ & $\circ$ & $\circ$ \\
\hline Gelati o granite (1 bicchiere/coppetta) & $\circ$ & $\circ$ & $\circ$ & $\circ$ & $\circ$ & $\circ$ & $\circ$ & $\circ$ & $\circ$ \\
\hline Cioccolata al latte (4 quadratini) & $\circ$ & $\circ$ & $\circ$ & $\circ$ & $\circ$ & $\circ$ & $\circ$ & $\circ$ & $\circ$ \\
\hline Cioccolata fondente (4 quadratini) & $\circ$ & $\circ$ & $\circ$ & $\circ$ & $\circ$ & $\circ$ & $\circ$ & $\circ$ & $\circ$ \\
\hline Patatine o altri snack salati (1 pacchetto) & $\circ$ & $\circ$ & $\circ$ & $\circ$ & $\circ$ & $\circ$ & $\circ$ & $\circ$ & $\circ$ \\
\hline Castagne (6 castagne) & $\circ$ & $\circ$ & $\circ$ & $\circ$ & $\circ$ & $\circ$ & $\circ$ & $\circ$ & $\circ$ \\
\hline Arachidi (10 arachidi) & $\circ$ & $\circ$ & $\circ$ & $\circ$ & $\circ$ & $\circ$ & $\circ$ & $\circ$ & $\circ$ \\
\hline Pistacchi (10 pistacchi) & $\circ$ & $\circ$ & $\circ$ & $\circ$ & $\circ$ & $\circ$ & $\circ$ & $\circ$ & $\circ$ \\
\hline Noci (5 noci) & $\circ$ & $\circ$ & $\circ$ & $\circ$ & $\circ$ & $\circ$ & $\circ$ & $\circ$ & $\circ$ \\
\hline Mandorle (10 mandorle) & $\circ$ & $\circ$ & $\circ$ & $\circ$ & $\circ$ & $\circ$ & $\circ$ & $\circ$ & $\circ$ \\
\hline Nocciole (10 nocciole) & $\circ$ & $\circ$ & $\circ$ & $\circ$ & $\circ$ & $\circ$ & $\circ$ & $\circ$ & $\circ$ \\
\hline Confetture, marmellate, miele (2 cucchiaini) & $\circ$ & $\circ$ & $\circ$ & $\circ$ & $\circ$ & $\circ$ & $\circ$ & $\circ$ & $\circ$ \\
\hline Creme spalmabili (nutella) (2 cucchiaini) & $\circ$ & $\circ$ & $\circ$ & $\circ$ & $\circ$ & $\circ$ & $\circ$ & $\circ$ & $\circ$ \\
\hline
\end{tabular}

\section{CONDIMENTI}

Sale aggiunto durante COTTURA

Sale aggiunto DOPO la cottura

Zucchero aggiunto a caffè o tè

Dolcificanti

Olio di oliva (1 cucchiaio)

Olio di semi (1 cucchiaio)

\begin{tabular}{|c|c|c|c|}
\hline \multicolumn{4}{|c|}{ Frequenze } \\
\hline$\circ$ MAI & o a volte & o spesso & o sempre \\
\hline ○ MAI & o a volte & o spesso & o sempre \\
\hline$\circ$ MAI & ○ mezzo cucchiaino & $\circ 1$ cucchiaino & $\circ 2$ cucchiaini \\
\hline$\circ$ MAI & ○ 1 goccia/compressa & $\circ 2$ gocce/compresse & - \\
\hline$\circ \mathrm{MAI}$ & $\circ$ meno di 1 volta al giorno & $\circ 1$ volta al giorno & $\circ 2$ o più volte al giorno \\
\hline$\circ$ MAI & o meno di 1 volta al giorno & ○ 1 volta al giorno & $\circ 2$ o più volte al giorno \\
\hline
\end{tabular}




\begin{tabular}{|c|c|c|c|c|c|c|c|c|c|}
\hline \multirow[b]{2}{*}{ VERDURA } & \multirow[b]{2}{*}{ Mai } & \multicolumn{2}{|c|}{ AL MESE } & \multicolumn{3}{|c|}{ A SETTIMANA } & \multicolumn{3}{|c|}{ AL GIORNO } \\
\hline & & $\begin{array}{c}1 \\
\text { al mese }\end{array}$ & $\begin{array}{c}2 \\
\text { al mese }\end{array}$ & $\begin{array}{c}1 \\
\text { a sett }\end{array}$ & $\begin{array}{l}2-3 \\
\text { a sett }\end{array}$ & $\begin{array}{l}4-5 \\
\text { a sett }\end{array}$ & $\begin{array}{c}1 \\
\text { al giorno }\end{array}$ & $\begin{array}{c}2-3 \\
\text { al giorno }\end{array}$ & $\begin{array}{c}4-5 \\
\text { al giorno }\end{array}$ \\
\hline Carota media (1 carota) & ○ & 0 & O & O & 0 & O & O & 0 & O \\
\hline Spinaci (120g) & 0 & 0 & 0 & 0 & 0 & 0 & 0 & 0 & 0 \\
\hline Broccoli (100g) (STAGIONE) & 0 & 0 & 0 & 0 & 0 & 0 & 0 & 0 & 0 \\
\hline Cavoli, cavolini di Bruxell (100g) (STAGIONE) & 0 & 0 & 0 & 0 & 0 & 0 & 0 & 0 & 0 \\
\hline Cavolfiore (70g) (STAGIONE) & 0 & 0 & 0 & 0 & 0 & 0 & 0 & 0 & 0 \\
\hline Piselli (100g) & 0 & 0 & 0 & 0 & 0 & 0 & 0 & 0 & 0 \\
\hline Fagiolini (150g) (STAGIONE) & 0 & 0 & 0 & 0 & 0 & 0 & 0 & 0 & 0 \\
\hline Zucchine $(100 \mathrm{~g})$ & 0 & 0 & 0 & 0 & 0 & 0 & 0 & 0 & 0 \\
\hline Cicoria $(120 \mathrm{~g})$ & 0 & o & O & 0 & 0 & 0 & 0 & 0 & 0 \\
\hline Asparagi (100g) (STAGIONE) & 0 & 0 & O & 0 & 0 & O & O & O & O \\
\hline Cipolla (4 fettine) & 0 & 0 & 0 & 0 & 0 & 0 & 0 & 0 & 0 \\
\hline Aglio (1 spicchio) & 0 & 0 & 0 & 0 & 0 & 0 & 0 & 0 & 0 \\
\hline Funghi (150g) (STAGIONE) & 0 & 0 & 0 & 0 & 0 & 0 & 0 & 0 & 0 \\
\hline Insalata verde, lattuga (100g) & 0 & 0 & 0 & 0 & 0 & 0 & 0 & 0 & 0 \\
\hline Pomodori (100g) & 0 & 0 & O & O & O & O & O & O & 0 \\
\hline Finocchi (mezzo) & 0 & 0 & O & 0 & 0 & O & 0 & O & O \\
\hline Mais $(100 \mathrm{~g})$ & 0 & O & O & O & O & O & 0 & O & O \\
\hline Fagioli $(100 \mathrm{~g})$ & 0 & 0 & 0 & 0 & 0 & 0 & 0 & 0 & 0 \\
\hline Ceci $(100 \mathrm{~g})$ & 0 & 0 & 0 & 0 & 0 & 0 & 0 & 0 & 0 \\
\hline Lenticchie (100g) & 0 & 0 & 0 & 0 & 0 & 0 & 0 & 0 & O \\
\hline Fave $(100 \mathrm{~g})$ & 0 & O & O & O & O & O & O & O & 0 \\
\hline Carciofi (1 carciofo) (STAGIONE) & 0 & 0 & 0 & 0 & 0 & 0 & 0 & O & 0 \\
\hline Soia, hamburger vegetali, tofu $(100 \mathrm{~g})$ & 0 & 0 & O & O & O & O & O & O & O \\
\hline Peperoni (1 peperone) & 0 & 0 & O & 0 & 0 & 0 & 0 & 0 & O \\
\hline Melanzana media (mezza melanzana) & 0 & 0 & 0 & 0 & 0 & 0 & 0 & 0 & 0 \\
\hline Olive verdi (4 olive) & 0 & 0 & 0 & 0 & 0 & 0 & 0 & 0 & 0 \\
\hline Olive nere (4 olive) & 0 & 0 & 0 & 0 & 0 & 0 & 0 & 0 & 0 \\
\hline
\end{tabular}

\begin{tabular}{|c|c|c|c|c|c|c|c|c|c|}
\hline \multirow[b]{2}{*}{ FRUTTA } & \multirow[b]{2}{*}{ Mai } & \multicolumn{2}{|c|}{ AL MESE } & \multicolumn{3}{|c|}{ A SETTIMANA } & \multicolumn{3}{|c|}{ AL GIORNO } \\
\hline & & $\begin{array}{c}1 \\
\text { al mese }\end{array}$ & $\begin{array}{c}2 \\
\text { al mese }\end{array}$ & $\begin{array}{c}1 \\
\text { a sett }\end{array}$ & $\begin{array}{l}2-3 \\
\text { a sett }\end{array}$ & $\begin{array}{l}4-5 \\
\text { a sett }\end{array}$ & $\begin{array}{c}1 \\
\text { al giorno }\end{array}$ & $\begin{array}{c}2-3 \\
\text { al giorno }\end{array}$ & $\begin{array}{c}4-5 \\
\text { al giorno }\end{array}$ \\
\hline Mela (1 frutto) & O & o & 0 & O & O & o & o & o & o \\
\hline Pera (1 frutto) & 0 & O & o & 0 & 0 & O & 0 & 0 & o \\
\hline Agrumi: arance, mandarini (1 frutto) (STAGIONE) & O & O & o & O & o & O & 0 & O & 0 \\
\hline Arancia rossa (1 frutto) (STAGIONE) & o & o & o & o & o & o & o & o & o \\
\hline Banana (1 frutto) & o & O & o & O & o & o & o & o & o \\
\hline Uva (6 chicchi) (STAGIONE) & o & 0 & o & o & o & o & o & o & 0 \\
\hline Melone (1 fetta) (STAGIONE) & o & O & o & o & o & o & o & o & o \\
\hline Anguria (1 fetta) (STAGIONE) & 0 & o & o & 0 & o & o & o & 0 & o \\
\hline Pesche (1 frutto) (STAGIONE) & O & 0 & O & 0 & 0 & 0 & 0 & 0 & 0 \\
\hline Albicocche (1 frutto) (STAGIONE) & 0 & 0 & 0 & 0 & 0 & 0 & 0 & 0 & 0 \\
\hline Fragole (4 fragole) (STAGIONE) & o & 0 & o & 0 & o & 0 & 0 & O & o \\
\hline Kiwi (1 frutto) (STAGIONE) & o & 0 & o & 0 & 0 & 0 & 0 & 0 & o \\
\hline Ciliegie (6 ciliegie) (STAGIONE) & o & O & o & o & o & o & o & o & o \\
\hline Frutti rossi: Mirtilli, gelsi e lamponi (STAGIONE) & o & o & o & 0 & o & o & o & 0 & o \\
\hline Melograno (1 frutto) (STAGIONE) & O & O & O & 0 & O & O & 0 & O & o \\
\hline Fichi d'india (1 frutto) (STAGIONE) & o & 0 & 0 & 0 & 0 & 0 & o & o & 0 \\
\hline Prugne, susine (1 frutto) (STAGIONE) & o & O & o & 0 & o & 0 & o & o & o \\
\hline Fichi (2 fichi) (STAGIONE) & o & O & 0 & 0 & 0 & 0 & 0 & 0 & 0 \\
\hline
\end{tabular}




\begin{tabular}{|c|c|c|c|c|c|c|c|c|c|}
\hline \multirow[b]{2}{*}{ BEVANDE } & \multirow[b]{2}{*}{ Mai } & \multicolumn{2}{|c|}{ AL MESE } & \multicolumn{3}{|c|}{ A SETTIMANA } & \multicolumn{3}{|c|}{ AL GIORNO } \\
\hline & & $\begin{array}{c}1 \\
\text { al mese }\end{array}$ & $\begin{array}{c}2 \\
\text { al mese }\end{array}$ & $\begin{array}{c}1 \\
\text { a sett }\end{array}$ & $\begin{array}{l}2-3 \\
\text { a sett }\end{array}$ & $\begin{array}{l}4-5 \\
\text { a sett }\end{array}$ & $\begin{array}{c}1 \\
\text { al giorno }\end{array}$ & $\begin{array}{c}2-3 \\
\text { al giorno }\end{array}$ & $\begin{array}{r}4-5 \\
\text { al giorno }\end{array}$ \\
\hline Thè nero o tisane (1 tazza) (NON alla frutta) & O & 0 & 0 & 0 & 0 & 0 & 0 & 0 & 0 \\
\hline Thè verde & O & 0 & o & 0 & 0 & 0 & 0 & 0 & 0 \\
\hline Thè, tisane $\mathrm{o}$ infusi alla frutta & O & O & O & O & O & O & O & O & O \\
\hline Caffè o cappuccino (1 tazza) & o & O & o & o & o & 0 & 0 & 0 & 0 \\
\hline Caffè o cappuccino DECAFFEINATO (1 tazza) & o & o & O & o & o & o & o & o & O \\
\hline Vino rosso (mezzo bicchiere) & 0 & 0 & o & 0 & 0 & 0 & 0 & 0 & 0 \\
\hline Vino bianco (mezzo bicchiere) & O & 0 & O & 0 & 0 & 0 & 0 & 0 & 0 \\
\hline Birra (1 birra piccola) & O & 0 & 0 & 0 & 0 & 0 & 0 & O & 0 \\
\hline Liquori (1 bicchierino) & 0 & 0 & o & 0 & 0 & 0 & 0 & 0 & 0 \\
\hline Bevande gassate (1 bicchiere) & O & O & 0 & 0 & 0 & 0 & 0 & 0 & 0 \\
\hline Spremute o frullati (frutta fresca) & O & O & O & O & O & O & O & O & O \\
\hline Spremute o frullati (CONFEZIONATI) & 0 & 0 & 0 & 0 & 0 & 0 & 0 & 0 & 0 \\
\hline Acqua (bicchieri al giorno) & $\begin{array}{c}\leq 3 \\
(600 \mathrm{ml})\end{array}$ & 4 & 5 & $\begin{array}{c}6 \\
(1,2 \mathrm{It}) \\
\end{array}$ & 7 & $\begin{array}{c}8 \\
(1,6 \mathrm{It}) \\
\end{array}$ & 9 & 10 & $\begin{array}{c}\geq 11 \\
(>2 \mathrm{It})\end{array}$ \\
\hline
\end{tabular}

\begin{tabular}{|c|c|c|c|c|c|c|c|c|c|}
\hline \multirow[b]{2}{*}{ CEREALI E FARINACEI } & \multirow[b]{2}{*}{ Mai } & \multicolumn{2}{|c|}{ AL MESE } & \multicolumn{3}{|c|}{ A SETTIMANA } & \multicolumn{3}{|c|}{ AL GIORNO } \\
\hline & & $\begin{array}{c}1 \\
\text { al mese }\end{array}$ & $\begin{array}{c}2 \\
\text { al mese }\end{array}$ & $\begin{array}{c}1 \\
\text { a sett }\end{array}$ & $\begin{array}{l}2-3 \\
\text { a sett }\end{array}$ & $\begin{array}{l}4-5 \\
\text { a sett }\end{array}$ & $\begin{array}{c}1 \\
\text { al giorno }\end{array}$ & $\begin{array}{c}2-3 \\
\text { al giorno }\end{array}$ & $\begin{array}{c}4-5 \\
\text { al giorno }\end{array}$ \\
\hline Pane e farinacei (fette biscottate, cracker) $(100 \mathrm{~g})$ & 0 & O & o & O & O & O & 0 & O & O \\
\hline Pane e farinacei INTEGRALI (100g) & 0 & 0 & 0 & 0 & 0 & 0 & 0 & 0 & 0 \\
\hline Corn flakes, cereali di frumento (30g) & 0 & 0 & 0 & 0 & 0 & 0 & 0 & 0 & 0 \\
\hline Riso $(80 \mathrm{~g})$ & 0 & 0 & 0 & 0 & 0 & 0 & 0 & 0 & 0 \\
\hline Pasta $(80 \mathrm{~g})$ & 0 & 0 & 0 & 0 & 0 & 0 & 0 & 0 & 0 \\
\hline Riso o pasta INTEGRALE (80g) & $\mathrm{O}$ & 0 & 0 & 0 & 0 & 0 & 0 & 0 & 0 \\
\hline Patate fritte $(100 \mathrm{~g})$ & 0 & 0 & 0 & 0 & 0 & 0 & 0 & 0 & 0 \\
\hline Patate bollite, al forno, purè (100g) & 0 & 0 & 0 & 0 & 0 & 0 & 0 & 0 & 0 \\
\hline Pizza (mezza pizza) & 0 & 0 & 0 & 0 & 0 & 0 & 0 & 0 & 0 \\
\hline Tavola calda (arancini, cipolline) & 0 & 0 & 0 & 0 & 0 & 0 & 0 & 0 & 0 \\
\hline
\end{tabular}

\begin{tabular}{|c|c|c|c|c|c|c|c|c|c|}
\hline \multirow[b]{2}{*}{ LATTE E DERIVATI } & \multirow[b]{2}{*}{ Mai } & \multicolumn{2}{|c|}{ AL MESE } & \multicolumn{3}{|c|}{ A SETTIMANA } & \multicolumn{3}{|c|}{ AL GIORNO } \\
\hline & & $\begin{array}{c}1 \\
\text { al mese }\end{array}$ & $\begin{array}{c}2 \\
\text { al mese }\end{array}$ & $\begin{array}{c}1 \\
\text { a sett }\end{array}$ & $\begin{array}{l}2-3 \\
\text { a sett }\end{array}$ & $\begin{array}{l}4-5 \\
\text { a sett }\end{array}$ & $\begin{array}{c}1 \\
\text { al giorno }\end{array}$ & $\begin{array}{c}2-3 \\
\text { al giorno }\end{array}$ & $\begin{array}{l}4-5 \\
\text { al giorno }\end{array}$ \\
\hline Latte (1 tazza, 250ml) & o & o & o & 0 & o & 0 & o & o & o \\
\hline Latte di soia, di riso (1 tazza, 250ml) & O & 0 & 0 & 0 & 0 & 0 & O & O & O \\
\hline Yogurt (1 vasetto) & O & o & o & o & o & o & o & o & o \\
\hline Formaggio giallo: Cheddar, Brie, caciotta, grana (50g) & 0 & 0 & 0 & 0 & 0 & 0 & 0 & 0 & 0 \\
\hline $\begin{array}{l}\text { Ricotta, formaggio a pasta molle basso contenuto di } \\
\text { grassi }(50 \mathrm{~g})\end{array}$ & O & ○ & ○ & ○ & ○ & o & ○ & $\circ$ & o \\
\hline Mozzarella (1 mozzarella) & o & o & o & o & o & 0 & o & o & o \\
\hline Formaggio spalmabile (Philadelfia) (30g) & ○ & O & O & 0 & 0 & 0 & O & 0 & 0 \\
\hline Burro (1 noce, 10g) & o & o & o & o & o & o & o & o & 0 \\
\hline Margarina (1 noce, $10 \mathrm{~g}$ ) & o & o & o & 0 & o & o & o & o & o \\
\hline
\end{tabular}

\begin{tabular}{|c|c|c|c|c|c|c|c|c|c|}
\hline \multirow[b]{2}{*}{ Integratori Alimentari o di erboristeria } & \multirow[b]{2}{*}{ Mai } & \multicolumn{2}{|c|}{ AL MESE } & \multicolumn{3}{|c|}{ A SETTIMANA } & \multicolumn{3}{|c|}{ AL GIORNO } \\
\hline & & $\begin{array}{c}1 \\
\text { al mese }\end{array}$ & $\begin{array}{c}2 \\
\text { al mese }\end{array}$ & $\begin{array}{c}1 \\
\text { a sett }\end{array}$ & $\begin{array}{l}2-3 \\
\text { a sett }\end{array}$ & $\begin{array}{l}4-5 \\
\text { a sett }\end{array}$ & $\begin{array}{c}1 \\
\text { al giorno }\end{array}$ & $\begin{array}{c}2-3 \\
\text { al giorno }\end{array}$ & $\begin{array}{c}4-5 \\
\text { al giorno }\end{array}$ \\
\hline Nome o tipo: & 0 & 0 & 0 & o & 0 & o & 0 & 0 & 0 \\
\hline Nome o tipo: & o & O & o & O & O & o & O & 0 & o \\
\hline
\end{tabular}

